\title{
Bone marrow fibrosis in patients with primary myelodysplastic syndromes has prognostic value using current therapies and new risk stratification systems
}

\author{
Bin $\mathrm{Fu}^{1,2,4}$, Jesse $\mathrm{M}$ Jaso $^{1}$, Rachel L Sargent ${ }^{1}$, Maitrayee Goswami ${ }^{1}$, Srdan Verstovsek $^{3}$,
} L Jeffrey Medeiros ${ }^{1}$ and Sa A Wang ${ }^{1}$

${ }^{1}$ Department of Hematopathology, The University of Texas MD Anderson Cancer Center, Houston, TX, USA;

${ }^{2}$ Department of Hematology, Central South University Xiangya Hospital, Changsha, China and ${ }^{3}$ Department of Leukemia, The University of Texas MD Anderson Cancer Center, Houston, TX, USA

\begin{abstract}
Bone marrow fibrosis has recently been recognized as an adverse histological feature in patients with primary myelodysplastic syndromes. In this study, we assessed the prognostic impact of bone marrow fibrosis in patients with primary myelodysplastic syndromes under the recently revised new risk stratification systems: the New Comprehensive Cytogenetic Scoring System and the Revised International Prognostic Scoring System. From 2002 to 2012, a total of 79 (13\%) patients with primary myelodysplastic syndromes and moderate/severe bone marrow fibrosis were identified; and these patients were compared with a control group of 166 patients with myelodysplastic syndromes but no significant fibrosis. Bone marrow fibrosis predicted an inferior overall survival and leukemia event-free survival for patients who received no hematopoietic stem cell transplant in univariate and multivariate analysis. Eleven patients with bone marrow fibrosis and 32 control group patients underwent hematopoietic stem cell transplant; and bone marrow fibrosis was an independent risk for an inferior overall survival but not leukemia-free survival. In addition, $17(4 \%)$ patients developed bone marrow fibrosis during the course of myelodysplastic syndromes, which was accompanied by clinical and cytogenetic evidence of disease progression. JAK2 V617F mutations were detected in 6 of the 28 patients with bone marrow fibrosis presenting at the time of diagnosis and 2 of the 7 patients with bone marrow fibrosis developing in the course of disease, significantly higher than the control group patients. We conclude that bone marrow fibrosis is an adverse risk feature in primary myelodysplastic syndromes in the current therapeutic era, and this risk feature is not captured by newly revised risk stratification systems. Inclusion of bone marrow fibrosis in patient assessment may further aid in risk-adapted therapeutic decisions.

Modern Pathology (2014) 27, 681-689; doi:10.1038/modpathol.2013.187; published online 1 November 2013
\end{abstract}

Keywords: bone marrow fibrosis; cytogenetics; JAK2 V617F; myelodysplastic syndromes; survival

Bone marrow fibrosis is observed in approximately $10-20 \%$ of patients with primary myelodysplastic syndromes. ${ }^{1,2}$ The clinical importance of bone marrow fibrosis in patients with myelodysplastic

Correspondence: Dr SA Wang, MD, Department of Hematopathology, The University of Texas MD Anderson Cancer Center, 1515 Holcombe Boulevard, Unit 72, Houston, TX 77030, USA.

E-mail: swang5@mdanderson.org

${ }^{4}$ The author conducted this study as a visiting scholar at The University of Texas MD Anderson Cancer Center, Houston, TX, USA.

Received 7 June 2013; revised 9 August 2013; accepted 27 August 2013; published online 1 November 2013 syndromes, however, has not been well recognized in the past and this histological feature was not incorporated into the 2008 World Health Organization classification system. ${ }^{3}$ In recent years, bone marrow fibrosis has been increasingly recognized as an adverse feature in patients with myelodysplastic syndromes, which correlates with increased risk of early bone marrow failure, transformation to acute myeloid leukemia, and an inferior patient outcome. ${ }^{4-8}$ Some of these studies, however, preceded the European consensus criteria established for grading bone marrow fibrosis ${ }^{9}$ and/or prior to the recent advent of therapeutic agents for myelodysplastic syndromes, such as lenolidomide 
and hypomethylating agents. In a study conducted by Della Porta et $a l^{5}$ patients with myelodysplastic syndromes who received hematopoietic stem cell transplantation or chemotherapy were censored at the time of therapeutic procedure. Therefore, the effect of bone marrow fibrosis in the current therapeutic paradigms is not clear. Furthermore, the molecular genetic features of these cases were not well described. Most importantly, the New Comprehensive Cytogenetic Scoring System for myelodysplastic syndromes and oligoblastic acute myeloid leukemia and the International Prognostic Scoring System-Revised have been published recently. ${ }^{10,11}$ These systems have been shown to improve risk stratification for patients with primary myelodysplastic syndromes. It is unknown if bone marrow fibrosis remains to be a risk factor in myelodysplastic syndromes stratified according to these new systems.

In this study, we retrospectively analyzed the clinicopathological features of a large cohort of patients with myelodysplastic syndromes with bone marrow fibrosis. Many of our patients were treated with hypomethylating agents and a subset of patients was treated with hematopoietic stem cell transplant. The goals of this study were two-fold. First, to investigate the clinicopathological, cytogenetic, and molecular genetic features of myelodysplastic syndromes with bone marrow fibrosis; and second, to examine the prognostic impact of bone marrow fibrosis in patients treated using current treatment modalities and stratified according to the recently revised New Comprehensive Cytogenetic Scoring System and Revised International Prognostic Scoring System systems.

\section{Materials and methods}

\section{Patients}

We reviewed the medical charts of all patients with a diagnosis of myelodysplastic syndromes at our hospital during a 10-year time interval (January 2003 through December 2012). The inclusion and exclusion criteria were as follows: (1) All cases met the diagnostic criteria for myelodysplastic syndromes according to the 2008 World Health Organization criteria, ${ }^{3}$ and the diagnosis was further confirmed in conjunction with clinical follow-up data; (2) bone marrow biopsy specimens at time of initial diagnosis of myelodysplastic syndromes were available for assessment of fibrosis; and (3) patients with a history or a suspected history of myeloproliferative neoplasms, myelodysplastic/ myeloproliferative neoplasm or patients who had therapy-related myelodysplastic syndromes were excluded. The study was conducted in accordance with the Declaration of Helsinki and the regulations of the Internal Review Board at MD Anderson Cancer Center.

\section{Bone Marrow Assessment}

Reticulin and trichrome stains were performed on bone marrow biopsy specimens in which fibrosis was suspected on initial evaluation of hematoxylineosin-stained slides based on the following features: increased stromal cells, cellular streaming/crushing, scarring/scleredema, dilated sinuses, or osteosclerosis. These features used for initial screening were based on our experience in myeloproliferative neoplasms, of which reticulin and trichrome are routinely performed in all cases. In total, reticulin/ trichrome stains were performed in $30-40 \%$ cases included in this study. Bone marrow fibrosis was graded according to the European bone marrow Fibrosis Network criteria ${ }^{12}$ as MF $0,1,2$, and 3. In some cases, bone marrow fibrosis was focal, ranging from MF0 to focal MF2 (often $<1 / 3$ of the areas), these cases were considered as $<\mathrm{MF} 2$ fibrosis. Only cases with $\geq \mathrm{MF} 2$ were considered to be moderate/ severe bone marrow fibrosis, henceforth, designated myelodysplastic syndromes with bone marrow fibrosis for this study. Bone marrow cellularity was estimated after excluding background fibrosis. Myeloblasts were enumerated as the percentage of total bone marrow nucleated cells. In cases of a 'dry tap', a CD34 immunohistochemical study was performed on the bone marrow biopsy specimens, and CD $34+$ blasts were counted/estimated under oil lens after excluding endothelial cells. Figure 1 illustrates a typical case of myelodysplastic syndromes with bone marrow fibrosis, with MF-2 fibrosis and $5-9 \%$ myeloblasts.

\section{Cytogenetic Classifications}

Conventional cytogenetic analysis was performed using the standard methods as described previously. ${ }^{13}$ Twenty metaphases were analyzed if available, and the results were reported using the International System for Human Cytogenetic Nomenclature. In some cases with a lesser number of metaphases available, fluorescence in situ hybridization was performed in order to confirm clonal cytogenetic abnormalities. Overall, we only included karyotype information for patients with adequate metaphases for analysis. The cytogenetic risk score was assigned to each patient following the New Comprehensive Cytogenetic Scoring System for primary myelodysplastic syndromes and oligoblastic acute myeloid leukemia and the International Prognostic Scoring System-Revised grouping criteria. ${ }^{10,11}$

\section{Molecular Analysis}

Molecular studies were performed using bone marrow aspirates. NPM1 mutations were detected using primers designed to amplify mutational hotspots spanning codons 956-971 of exon 12, followed 




Figure 1 Bone marrow features of myelodysplastic syndromes with moderate-to-severe bone marrow fibrosis. (a) Hematoxylin and eosin $($ H\&E magnification $\times 500$ ) showing a hypercellular bone marrow with markedly increased dysplastic megakaryocytes; (b) reticulin stain $(\times 500)$ showing markedly increased reticulin fibrosis; (c) CD34 immunohistochemistry $(\times 500)$ showing increased small vessels as well as CD34 + myeloblasts; (d) paucicellular bone marrow aspirate (due to bone marrow fibrosis) showing dysplastic neutrophils and erythroid precursors.

by capillary electrophoresis as described previously. ${ }^{14}$ The FLT3 internal tandem duplication (FLT3-ITD) and tyrosine kinase domain codon 835/ 836 point mutations (FLT3-D835) were detected by a fluorescent-based multiplex PCR assay followed by capillary electrophoresis. ${ }^{14}$ For FLT3-D835 point mutation analysis, PCR products were digested with EcoRV before capillary electrophoresis. MPL, JAK2 V617F, K-RAS, and N-RAS mutations at codons 515, 617, 13, and 61 were tested using PCR followed by pyrosequencing as described previously. ${ }^{15}$ Mutations in exons 8 and 17 of the KIT gene were detected using Sanger sequencing. ${ }^{16}$

Treatment for Myelodysplastic Syndrome and Followup Bone Marrow Evaluation

Therapies received by patients with myelodysplastic syndromes were grouped as follows: best supportive care; induction chemotherapy; low-intensity chemo- therapy, hypomethylating agents; immunomodulatory therapy (thalidomide/lenalidomide, investigational drugs, and immunosuppressive agents); and hematopoietic stem cell transplant. If a patient had received more than one treatment category, the patient was assigned to the more intensive treatment category. In patients with bone marrow fibrosis at time of diagnosis, bone marrow fibrosis was reassessed in patients with follow-up bone marrow biopsy specimens, if available.

\section{Statistical Analyses}

For continuous variables, data were reported as medians and ranges and compared by the MannWhitney $U$-test. Fisher's exact test or $\chi^{2}$ were used for categorical comparisons. Overall survival and leukemia-free survival were estimated by the Kaplan-Meier method and log-rank test. Multivariate analysis was performed using the Cox regression 
model with categorical variables. All $P$ values were two-tailed and were considered significant when $<0.05$.

\section{Results}

Clinicopathological Findings in Myelodysplastic Syndrome Patients with Bone Marrow Fibrosis at Initial Diagnosis

We identified 630 patients diagnosed during the study interval with primary/de novo myelodysplastic syndromes who had initial bone marrow aspirate smears and biopsy specimens available for assessment. Substantial bone marrow fibrosis (grade MF2 and MF3) was found in 79 patients (13\%). From the remaining patients with no significant bone marrow fibrosis (<MF2), we randomly chose one-third $(n=166)$ for use as a control group. The demographic and hematological data and cytogenetic features of these patients are summarized in Table 1. In brief, patients with bone marrow fibrosis had a comparable age but less pronounced male predominance comparing with control group of patients. Hepatosplenomegaly, consumptive symptoms, and transfusion dependency were more commonly observed in patients with bone marrow fibrosis $(P=0.079, P=0.002$, and $P<0.001$, respectively). A comparison of the complete blood count data showed that the leukocyte count, absolute neutrophil count, and hemoglobin levels were comparable between patients with or without bone marrow fibrosis, but thrombocytopenia $(P<0.001)$ was more severe in the fibrotic group of patients. Patients with a fibrotic bone marrow had a borderline higher number of circulating blasts compared with the control group of patients $(P=0.068)$.

Comparing the bone marrow findings, cases with bone marrow fibrosis had a lower cellularity $(P=0.020)$ but a higher number of megakaryocytes $(P<0.001)$. Dysplasia, including dysmegakaryopoiesis, was similarily observed in cases with bone marrow fibrosis and controls, $89 \%$ vs $85 \%$, respectively $(P=0.214)$. The percentage of bone marrow blasts was comparable.

\section{Cytogenetic Features of Myelodysplastic Syndrome Patients with Bone Marrow Fibrosis}

Overall, conventional cytogenetic analysis was available/successful in 237 of the 245 (97\%) patients (Table 1), including 74/79 (94\%) patients with bone marrow fibrosis and 163/166 (98\%) patients in the non-fibrotic group. The median metaphases obtained for karyotype analysis were 20 (range, 530 ) in the fibrotic group and also 20 (range, 2-29) in the control group $(P=0.259)$. Cases of myelodysplastic syndromes with or without bone marrow fibrosis had a similar cytogenetic risk distribution using the New Comprehensive Cytogenetic Scoring
System for primary myelodysplastic syndromes/ oligoblastic acute myeloid leukemia ${ }^{11}$ and a similar frequency of a monosomal karyotype (ie, at least one chromosome showing monosomy and at least one structural abnormality; 24 vs $18 \%$, $P=0.190)$.

\section{Mutation Studies in Myelodysplastic Syndrome Patients with Bone Marrow Fibrosis}

Mutation studies for JAK2, RAS, FLT3, KIT, NPM1, $C E B P A$, and $M P L$ were performed in variable subsets of patients as a part of their routine work-up using whole-cell lysate of bone marrow aspitates. JAK2 V617F mutations were detected in 6/28 (21\%) patients with bone marrow fibrosis vs $1 / 41$ (2\%) patients without fibrosis, $(P=0.014)$. The median allele burden of mutant JAK2 $V 617 F$ was $22.5 \%$ (5.5-46\%). Of patients with bone marrow fibrosis, two patients with mutated JAK2 V617F vs $3 / 23$ patients with wildtype JAK2 V617F had organomegaly (not significant); megakaryocyte morphology, grouped as myelodysplastic type, myeloproliferative type or mixed, was not significantly different between cases with mutated and wild-type JAK2 $V 617 F$. The other genes assessed were detected at low frequency and did not show any significant differences between patients with bone marrow fibrosis vs patients without.

Specifically, RAS mutations were detected in $0 / 32$ $(0 \%)$ patients with bone marrow fibrosis and 4/140 (3\%) control patients. FLT3-ITD mutations were detected in $1 / 55(2 \%)$ patients with bone marrow fibrosis and in $0 / 142(0 \%)$ control patients. Mutations in KIT were detected in $0 / 29(0 \%)$ patients with bone marrow fibrosis vs $1 / 87(1 \%)$ control patients. NPM1 mutations were present in $2 / 24$ $(8 \%)$ patients with bone marrow fibrosis vs $2 / 71$ (3\%) control patients, Mutations of $M P L$ were found in $0 / 8(0 \%)$ patients with bone marrow fibrosis vs $0 /$ $2(0 \%)$ control patients. CEBPA mutations were observed in $0 / 19(0 \%)$ patients with bone marrow fibrosis vs $5 / 48(10 \%)$ control patients.

\section{Effect of Bone Marrow Fibrosis on Overall and Leukemia-Free Survival of Myelodysplastic Syndrome Patients}

For the subset of patients who did not receive hematopoietic stem cell transplant, bone marrow fibrosis was a predictor of inferior overall survival and leukemia-free survival (myeloproliferative neoplasms) by Kaplan-Meier estimate. Patients with bone marrow fibrosis who received no hematopoietic stem cell transplant had a median overall survival of 21 months (95\% CI, 16-26 months) and a leukemia-free survival of 52 months (95\% CI, 40-65 months), significantly worse than the control group of patients who had a median overall survival of 42 months (95\% CI, 29-55 months; log rank, $P=0.000$ ); 
Table 1 Clinicopathological and molecular genetic comparisons of patients with myelodysplastic syndromes with or without bone marrow fibrosis

\begin{tabular}{|c|c|c|c|}
\hline & \multicolumn{2}{|c|}{ Bone marrow fibrosis } & \multirow[b]{2}{*}{$P$} \\
\hline & $\begin{array}{l}M F 0-1 \\
(\mathrm{n}=166)\end{array}$ & $\begin{array}{l}M F 2-3 \\
(\mathrm{n}=79)\end{array}$ & \\
\hline Age (years) & $64(15-89)$ & $65(17-91)$ & 0.446 \\
\hline Gender (male/female) & $120 / 46$ & $44 / 35$ & 0.008 \\
\hline $\begin{array}{l}\text { Absolute neutrophil count } \\
\left(\times 10^{9 / 1}\right)\end{array}$ & $1.5(0-8.5)$ & $1.5(0.1-11.3)$ & 0.756 \\
\hline Hemoglobin level (g/dl) & $9.7(5.5-15.4)$ & $9.3(5.7-16.0)$ & 0.138 \\
\hline Platelets count $\left(\times 10^{9 / 1}\right)$ & $94(6-885)$ & $51(4-352)$ & 0.000 \\
\hline Bone marrow myeloblasts (\%) & $3.5(0-18 \%)$ & $3.5(0-19 \%)$ & 0.970 \\
\hline Peripheral myeloblasts (\%) & $0.2(0-11 \%)$ & $0.4(0-10 \%)$ & 0.068 \\
\hline BM cellularity (\%) & $72(5-100 \%)$ & $64(10-100 \%)$ & 0.020 \\
\hline \multicolumn{3}{|l|}{ Megakaryocvtes } & 0.000 \\
\hline Decreased $(\leq 1 / \mathrm{HPF})$ & $36(22 \%)$ & $11(14 \%)$ & \\
\hline Normal $(2-5 / \mathrm{HPF})$ & $61(37 \%)$ & $10(13 \%)$ & \\
\hline Increased ( $\geq 6 / \mathrm{HPF})$ & $67(41 \%)$ & $57(73 \%)$ & \\
\hline Hepatosplenomegaly & $9 / 161(6 \%)$ & $9 / 76(12 \%)$ & 0.079 \\
\hline Constitutional symptoms & 18/161 (11\%) & $20 / 71(28 \%)$ & 0.002 \\
\hline Transfusion dependency & $59 / 162(36 \%)$ & $16 / 45(62 \%)$ & 0.000 \\
\hline JAK2 V617 mutation & $1 / 41(2 \%)$ & $6 / 28(21 \%)$ & 0.014 \\
\hline \multicolumn{3}{|l|}{$\begin{array}{l}\text { World Health Organization } \\
\text { classification }\end{array}$} & 0.701 \\
\hline $5 q-$ syndromes & $6(4 \%)$ & 0 & \\
\hline $\begin{array}{l}\text { Refractory anemia; } \\
\text { refractory anemia with ring } \\
\text { sideroblasts; refractory } \\
\text { cytopenia with unilineage } \\
\text { dysplasia and } \\
\text { myelodysplastic } \\
\text { syndromes-unclassified }\end{array}$ & $30(18 \%)$ & $13(17 \%)$ & \\
\hline $\begin{array}{l}\text { Refractory cytopenia with } \\
\text { multilineage dysplasia }\end{array}$ & $64(39 \%)$ & $29(37 \%)$ & \\
\hline $\begin{array}{l}\text { Refractory anemia with } \\
\text { excess blasts-1 }\end{array}$ & $34(21 \%)$ & $19(24 \%)$ & \\
\hline $\begin{array}{l}\text { Refractory anemia with } \\
\text { excess blasts-2 }\end{array}$ & $32(19 \%)$ & $18(23 \%)$ & \\
\hline \multicolumn{3}{|l|}{$\begin{array}{l}\text { Cytogenetics by New } \\
\text { Comprehensive Cytogenetic }\end{array}$} & 0.163 \\
\hline Comprehensive Cytogenetic & & & \\
\hline \multicolumn{4}{|l|}{ Scoring System } \\
\hline Very good & $4 / 163(3 \%)$ & 0 & \\
\hline Good & $95 / 163(58 \%)$ & $37 / 76(49 \%)$ & \\
\hline Intermediate & $27 / 163(17 \%)$ & $13 / 76(17 \%)$ & \\
\hline Poor & $11 / 163(7 \%)$ & $11 / 76(15 \%)$ & \\
\hline Very poor & $26 / 163(16 \%)$ & $15 / 76(20 \%)$ & \\
\hline \multirow{2}{*}{\multicolumn{4}{|c|}{$\begin{array}{l}\text { Revised International } \\
\text { Prognostic Scoring System } \\
\text { risk categories }\end{array}$}} \\
\hline & & & \\
\hline Very low & $16(10 \%)$ & $4(5 \%)$ & \\
\hline Low & $54(33 \%)$ & $17(23 \%)$ & \\
\hline Intermediate & $40(25 \%)$ & $21(28 \%)$ & \\
\hline High & $26(16 \%)$ & $11(15 \%)$ & \\
\hline Very high & $27(17 \%)$ & $21(28 \%)$ & \\
\hline Leukemia transformation & $43 / 166(26 \%)$ & $29 / 79(37 \%)$ & 0.057 \\
\hline
\end{tabular}

and a leukemia-free survival of 120 months (95\% CI, 102-137 months; log rank, $P=0.003$ ).

In this patient cohort, 128 patients had $<5 \%$ bone marrow and peripheral blood blasts and 74 patients had $\geq 5 \%$ blasts in either bone marrow and/or peripheral blood. For the subset of patients with $<5 \%$ blasts, patients with bone marrow fibrosis $(n=44)$ had a median overall survival of 22 months (95\% CI, 15-29 months) and a leukemia-free survival of 62 months (95\% CI, 46-79 months) compared with patients without bone marrow fibrosis $(n=84)$ who had a median overall survival of 58 months (95\% CI, 26-90 months; log rank, $P<0.001)$ and a leukemia-free survival of 150 months (95\% CI, 135-166 months; log rank, $P=0.007$ ) (Figure 2a). For patients with $\geq 5 \%$ blasts, patients with bone marrow fibrosis $(n=24)$ had a median overall survival of 13 months (95\% CI, 9-17 months) vs control group patients $(n=50)$ who had a median overall survival of 21 months (95\% CI, 1428 months; log rank, $P=0.030$ ) (Figure 2b). A similar trend for leukemia-free survival was observed: 17 months (95\% CI, 9-25 months) in patients with bone marrow fibrosis vs 32 months (95\% CI, 14-50 months) in control patients; however, these results were not statistically significant (log rank, $P=0.204$ ).

The risk of bone marrow fibrosis was co-analyzed with other parameters in Cox regression multivariate analysis that included cytogenetic risk, age, gender, leukocyte count, absolute neutrophil count, platelet count, hemoglobin level, and bone marrow or peripheral blood blasts (Table 2). The presence of bone marrow fibrosis was an independent hazard for an inferior overall survival as well as leukemia-free survival in patients who were not treated with hematopoietic stem cell transplant.

For the subset of patients who were treated with hematopoietic stem cell transplant, 11 patients had bone marrow fibrosis and 32 patients had no or mild bone marrow fibrosis. The Kaplan-Meier estimate showed a trend for an inferior overall survival and leukemia-free survival in patients with bone marrow fibrosis; however, the small numbers of patients probably limited this analysis $(P=0.144$ and $P=0.092$ ). In the multivariate analysis, however, bone marrow fibrosis was an adverse risk factor for an inferior overall survival $(P=0.048)$ but not leukemia-free survival $(P=0.458)$.

\section{Response of Bone Marrow Fibrosis in Myelodysplastic Syndrome Patients Treated with Various Modalities}

In this study group, the treatment modalities administered to patients with or without bone marrow fibrosis were not significantly different. These modalities included: best supportive care (15/79 for patients with bone marrow fibrosis vs 39/ 166 controls), low intensity chemotherapy or immunomodulatory therapy (13/79 vs 17/166), hypomethylating agents (37/79 vs 73/166), induction chemotherapy (3/79 vs 5/166), and allogeneic hematopoietic stem cell transplant (11/79 vs 32/ 166). Comparing treatment responses to various therapeutic modalities in patients with vs without bone marrow fibrosis was difficult due to the natural history of myelodysplastic syndromes and the complexity of patient management. In this study, we focused our evaluation on patients with bone 

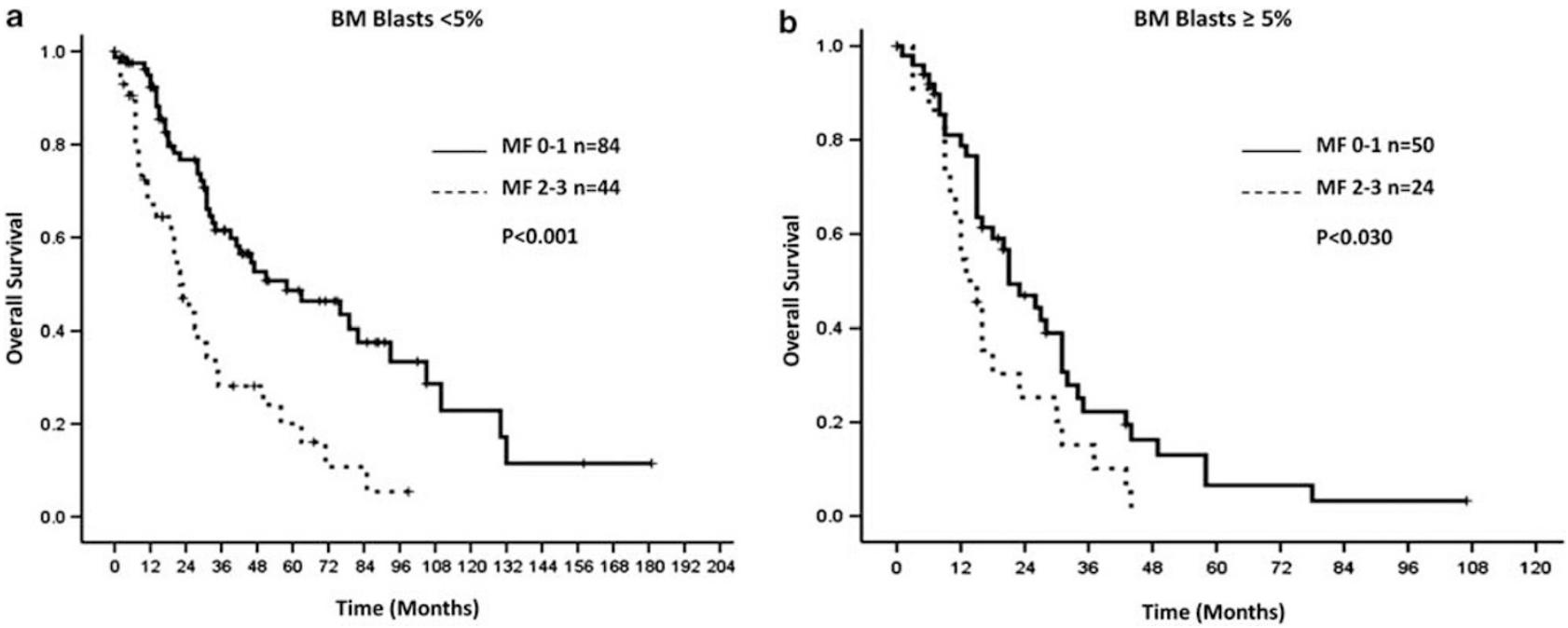

Figure 2 The effect of bone marrow fibrosis on overall survival of myelodysplastic syndrome patients who were not treated with hematopoietic stem cell transplant. The presence of bone marrow fibrosis is a significant predictor for overall survival in patients with $<5 \%$ (a) or $\geq 5 \%$ bone marrow blasts (b)

Table 2 Risk features of bone marrow fibrosis in patients with myelodysplastic syndromes by Cox regression multivariate analysis

\begin{tabular}{|c|c|c|c|c|}
\hline & \multicolumn{2}{|c|}{$\begin{array}{l}\text { Non-hematopoietic stem } \\
\text { cell transplantation }\end{array}$} & \multicolumn{2}{|c|}{$\begin{array}{l}\text { Hematopoietic stem } \\
\text { cell transplantation }\end{array}$} \\
\hline & $\operatorname{Exp}(B) 95.0 \% C I$ & $\mathrm{P}$ & $\operatorname{Exp}(B) 95.0 \% C I$ & $\mathrm{P}$ \\
\hline Age & $1.035(1.016-1.054)$ & $0.000^{\mathrm{a}}$ & $1.013(0.969-1.059)$ & $0.578^{\mathrm{a}}$ \\
\hline Absolute neutrophil count & $0.962(0.873-1.061)$ & $0.441^{\mathrm{a}}$ & $0.631(0.411-0.967)$ & $0.035^{\mathrm{a}}$ \\
\hline Platelets count & $0.999(0.997-1.000)$ & $0.129^{\mathrm{a}}$ & $1.004(1.000-1.008)$ & $0.036^{\mathrm{a}}$ \\
\hline Hemoglobin level & $0.838(0.745-0.943)$ & $0.003^{\mathrm{a}}$ & $1.062(0.772-1.461)$ & $0.712^{\mathrm{a}}$ \\
\hline & $0.866(0.774-0.969)$ & $0.012^{\mathrm{b}}$ & - & - \\
\hline Bone marrow blasts & $1.050(1.013-1.090)$ & $0.008^{\mathrm{a}}$ & $1.078(0.961-1.209)$ & $0.201^{\mathrm{a}}$ \\
\hline & $1.064(1.025-1.103)$ & $0.001^{\mathrm{b}}$ & - & - \\
\hline Cytogenetic risk by New Comprehensive & $1.463(1.227-1.743)$ & $0.000^{\mathrm{a}}$ & $3.978(2.074-7.630)$ & $0.000^{\mathrm{a}}$ \\
\hline Cytogenetic Scoring System & $1.477(1.244-1.753)$ & $0.000^{\mathrm{b}}$ & $2.776(1.720-4.479)$ & $0.000^{\mathrm{b}}$ \\
\hline Bone marrow myelofibrosis & $\begin{array}{l}1.732(1.154-2.600) \\
1.730(1.171-2.555)\end{array}$ & $\begin{array}{l}0.008^{\mathrm{a}} \\
0.006^{\mathrm{b}}\end{array}$ & $\begin{array}{r}3.213(0.873-11.831) \\
2.700(1.010-7.220)\end{array}$ & $\begin{array}{l}0.079^{\mathrm{a}} \\
0.048^{\mathrm{b}}\end{array}$ \\
\hline
\end{tabular}

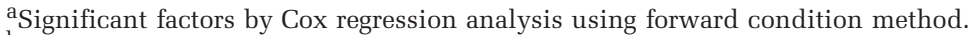

${ }^{\mathrm{b}}$ Significant factors by Cox regression analysis using enter method.

marrow fibrosis at the time when they achieved their best response to therapies. Follow-up bone marrow biopsy specimens were available in 60 of 79 patients with bone marrow fibrosis. For the subset of 27 patients with bone marrow fibrosis treated with hypomethylating agents, 3 patients achieved complete hematological response, 6 patients had stable disease, and 18 patients had disease progression. Only one patient showed reduced bone marrow fibrosis, but his disease progressed. For the subset of 11 patients with bone marrow fibrosis who received hematopoietic stem cell transplant, 4 patients had complete resolution of bone marrow fibrosis at the time of last follow-up. Three of these patients achieved complete hematological response and 1 patient experienced disease relapse. For the subset of 22 patients who received supportive care and other treatment, only 1 patient who received lenalidomide showed improvement of bone marrow fibrosis.

\section{Importance of Bone Marrow Fibrosis that Develops During the Course of Myelodysplastic Syndromes}

There were 551 patients with no significant bone marrow fibrosis at the time of diagnosis; 480 patients had follow-up bone marrow biopsy specimens available for review (median 4 bone marrow specimens). Seventeen (4\%) patients developed bone marrow fibrosis during the follow-up interval. There were 12 men and 5 women with a median age of 72 years (range, 51-83 years). The median interval from initial diagnosis to development of bone 
marrow fibrosis was 22 months (range, 2-126 months). The median patient survival after bone marrow fibrosis developed was 9 months (range, 2-22 months). None of these patients received hematopoietic stem cell transplant. At the time of bone marrow fibrosis development, 8 of the 17 patients showed evidence of disease progression, from low-risk categories $(5 q-$ syndrome, refractory cytopenia with unilineage or multilineage dysplasia) to refractory anemia with excess blasts (Table 3). Cytogenetic clonal evolution was found in 4 of the 16 patients. Three of these cases began as myelodysplastic syndromes with isolated del (5q) and acquired additional del (7q) or -7 at the time of onset of bone marrow fibrosis. None of these 17 patients had repeat mutation studies performed preand post-bone marrow fibrosis; therefore, molecular evolution associated with bone marrow fibrosis could not be assessed. At the time of bone marrow fibrosis, JAK2 V617 mutations were detected in 2/7 $(29 \%)$ patients and $R A S$ mutations in $1 / 10(10 \%)$. No patients tested had evidence of FLT3 $(n=12)$, NPM1 $(n=7)$, or MPL $(n=2)$ mutations.

\section{Discussion}

In this study consisting of the largest cohort of patients with primary myelodysplastic syndromes and bone marrow fibrosis, we show that bone marrow fibrosis occurred in $13 \%$ patients at the time of initial diagnosis. This frequency is similar to that reported previously of $10-20 \%{ }^{2,4-6}$ Bone marrow fibrosis in patients with primary myelodysplastic syndromes was more frequently associated with hepatosplenomegaly, consumptive symptoms, and transfusion

Table 3 Clinicopathological features of 17 patients who developed bone marrow fibrosis later in the course of myelodysplastic syndromes

\begin{tabular}{|c|c|c|c|}
\hline & $\begin{array}{c}\text { Initial } \\
\text { diagnosis }\end{array}$ & $\begin{array}{l}\text { Bone } \\
\text { marrow } \\
\text { fibrosis }\end{array}$ & $\mathrm{P}$ \\
\hline Transfusion dependency & $8 / 17$ & $9 / 17$ & 1.000 \\
\hline Cytogenetics & - & $4 / 16$ & \\
\hline Normal & $9 / 16$ & $8 / 17$ & 0.732 \\
\hline Del (5q) & $3 / 16$ & $3 / 17$ & \\
\hline$-7 / \mathrm{del}(7 \mathrm{q})$ & $0 / 16$ & $4 / 17$ & \\
\hline+8 & $1 / 16$ & $2 / 16$ & \\
\hline $\begin{array}{l}\text { The World Health Organization } \\
\text { categories }\end{array}$ & & & 0.069 \\
\hline $\begin{array}{l}\text { Refractory anemia; refractory anemia } \\
\text { with ring sideroblasts; refractory } \\
\text { cytopenia with unilineage dysplasia }\end{array}$ & 6 & 1 & \\
\hline $\begin{array}{l}\text { Refractory cytopenia with } \\
\text { multilineage dysplasia }\end{array}$ & 6 & 5 & \\
\hline $\begin{array}{l}\text { Refractory anemia with excess } \\
\text { blasts-1 }\end{array}$ & 2 & 7 & \\
\hline $\begin{array}{l}\text { Refractory anemia with excess } \\
\text { blasts-2 }\end{array}$ & 2 & 4 & \\
\hline
\end{tabular}

dependency. Bone marrow fibrosis was also accompanied by increased megakaryocytes and more severe thrombocytopenia, supporting the theory that megakaryocytic proliferation and the interaction between megakaryocytes and bone marrow stroma have a role in bone marrow fibrosis. ${ }^{17}$

Recent advances in myelodysplastic syndrome treatment, including hypomethylating agents and hematopoietic stem cell transplant, have improved patients' outcome. In this study, we showed that hypomethylating agents appeared to have no impact on improving bone marrow fibrosis, even for those patients who achieve complete hematological responses. Similar findings have been reported in patients with primary myelofibrosis that bone marrow fibrosis is not significantly altered in response to chemotherapy. ${ }^{18}$ In contrast, four of the 11 patients in this study who achieved complete hematological response or bone marrow complete hematological response after hematopoietic stem cell transplant showed complete resolution of bone marrow fibrosis at the time of last follow-up. ${ }^{19}$ It has been shown that in patients with bone marrow fibrosis related to myeloproliferative neoplasm, allogeneic stem cell transplantation resulted in a rapid resolution of bone marrow fibrosis, suggesting graft-anti-fibrosis effect. ${ }^{20}$

The results we present here confirm the prognostic importance of bone marrow fibrosis in patients with primary myelodysplastic syndromes. ${ }^{1,4-7}$ This adverse feature not only affected patients treated with non-transplant modalities but also patients who received hematopoietic stem cell transplant. The latter was in keeping with the study reported by Kroger et $a l^{19}$ that severe bone marrow fibrosis was an independent risk affecting transplant outcome in patients with myelodysplastic syndromes. Some previously published studies suggested that worse survivals observed might be partially due to poor risk cytogenetics associated with cases with bone marrow fibrosis. ${ }^{10,11,13}$ However, we showed that the distribution of cytogenetic risk stratified by the New Comprehensive Cytogenetic Scoring System was not significantly different between patients with or without bone marrow fibrosis, and that bone marrow fibrosis was a risk feature independent of cytogenetics. The World Health Organization categories for myelodysplastic syndromes, as well as Revised International Prognostic Scoring System scores, also showed no significant differences in risk distribution between myelodysplastic syndrome patients with or without bone marrow fibrosis. We therefore suggest that bone marrow fibrosis, which is not captured by the current risk classification/ stratification systems, should be used in the evaluation of myelodysplastic syndrome patients. Ideally, bone marrow fibrosis should be integrated into currently used risk classification/stratification systems.

In this study, we also identified 17 patients who had myelodysplastic syndromes without bone 
marrow fibrosis at initial diagnosis but subsequently developed bone marrow fibrosis over the course of their disease and died, usually in $<1$ year (median interval 9 months). The development of bone marrow fibrosis was accompanied by disease progression that could be measured by other parameters. Eight of the 17 patients showed increased bone marrow blasts from $<5 \%$ to $\geq 5 \%$, progressed to either refractory anemia with excess blasts or acute myeloid leukemia, and one quarter of the patients showed clonal cytogenetic evolution. Therefore, bone marrow fibrosis developing over the course of disease in patients with primary myelodysplastic syndromes may not be an independent risk factor but rather one of a constellation of features associated with disease progression.

In this study, we detected JAK2 V617F mutations in 6 of the $28(21 \%)$ myelodysplastic syndrome patients with bone marrow fibrosis at the time of diagnosis and in 2 of the 7 (29\%) patients who developed bone marrow fibrosis over the course of disease. Notably, the JAK2 V617 allele burden appeared to be lower than that of primary myelofibrosis (in our laboratory: median $47 \%, n=243$, unpublished data). Ohyashiki et $a l^{21}$ reported JAK2 $V 617 F$ mutations in 2 of the 6 patients who developed secondary bone marrow fibrosis in the course of myelodysplastic syndromes. Later studies have reported a variable rate of JAK2 V617F mutations in myelodysplastic syndromes with bone marrow fibrosis, ranging from 0 to $18 \% .^{4,22-25}$ Notably, these studies only tested small numbers of cases due to the unavailability of DNA. Nevertheless, based on these reports, JAK2 V617F mutation analysis has been suggested as a test that could discriminate myelodysplastic syndromes from myeloproliferative neoplasms in patients with bone marrow fibrosis. ${ }^{26}$ In this study, all cases of myelodysplastic syndromes with bone marrow fibrosis fulfilled the World Health Organization diagnostic criteria for myelodysplastic syndromes and had no documented preceding history of myeloproliferative neoplasms or hybrid hematological features of a myelodysplastic/myeloproliferative syndrome. These findings allow us to speculate that JAK2 V617F mutations may contribute to the development of bone marrow fibrosis in a subset of these patients. Sokol et a $2^{27}$ also suggested the possibility of multiclonality that a subclone of stem cells may acquire JAK2 V617F mutation in the course of myelodysplastic dyndromes. Interestingly, it has been known that patients with myelodysplastic syndromes are predominantly male; whereas, patients with Philadelphia chromosome-negative myeloproliferative neoplasms are predominantly female. ${ }^{28}$ We observed a much less pronounced male predominance in our patients with bone marrow fibrosis. Also, patients with bone marrow fibrosis showed more frequent organomegaly and consumptive symptoms; and their bone marrow showed megakaryocytic hyperplasia. Considered together, these findings support the notion that bone marrow fibrosis may be regarded as a proliferative feature; and the biological nature of myelodysplastic syndromes with bone marrow fibrosis may be more closely associated with myelodysplastic syndromes/myeloproliferative neoplasms. In summary, in this study we showed that bone marrow fibrosis at the time of initial diagnosis of myelodysplastic syndromes is an adverse histological feature that is not captured by the current World Health Organization classification or the recently revised New Comprehensive Cytogenetic Scoring System cytogenetic classification and Revised International Prognostic Scoring System risk stratification systems. Bone marrow fibrosis also appears to affect outcome of hematopoietic stem cell transplant. In $3-4 \%$ of patients, bone marrow fibrosis can develop over the course of disease and is often associated with disease progression. JAK2 V617F mutations are detected in approximately $20 \%$ patients with primary myelodysplastic syndromes. The clinicopathological features and molecular alterations suggest that myelodysplastic syndromes with bone marrow fibrosis may be more akin to myelodysplastic syndromes/myeloproliferative neoplasms than myelodysplastic syndromes.

\section{Disclosure/conflict of interest}

The authors declare no conflict of interest.

\section{Author contributions}

SAW and SV designed the study; BF, RLS, JMJ, MG and SAW conducted data retrieval; BF, LJM and SAW analyzed the data; and BF, SAW and LJM wrote the manuscript. All authors read and approved the final manuscript.

\section{References}

1 Verhoef GE, De Wolf-Peeters C, Ferrant A, et al. Myelodysplastic syndromes with bone marrow fibrosis: a myelodysplastic disorder with proliferative features. Ann Hematol 1991;63:235-241.

2 Lambertenghi-Deliliers G, Orazi A, Luksch R, et al. Myelodysplastic syndrome with increased marrow fibrosis: a distinct clinico-pathological entity. $\mathrm{Br} \mathrm{J}$ Haematol 1991;78:161-166.

3 Vardiman JW, Thiele J, Arber DA, et al. The 2008 revision of the World Health Organization (WHO) classification of myeloid neoplasms and acute leukemia: rationale and important changes. Blood 2009;114:937-951.

4 Buesche G, Teoman H, Wilczak W, et al. Marrow fibrosis predicts early fatal marrow failure in patients with myelodysplastic syndromes. Leukemia 2008;22:313-322.

5 Della Porta MG, Malcovati L, Boveri E, et al. Clinical relevance of bone marrow fibrosis and CD34-positive 
cell clusters in primary myelodysplastic syndromes. J Clin Oncol 2009;27:754-762.

6 Lambertenghi-Deliliers G, Annaloro C, Oriani A, et al. Prognostic relevance of histological findings on bone marrow biopsy in myelodysplastic syndromes. Ann Hematol 1993;66:85-91.

7 Maschek H, Georgii A, Kaloutsi V, et al. Myelofibrosis in primary myelodysplastic syndromes: a retrospective study of 352 patients. Eur J Haematol 1992;48: 208-214.

8 Maschek $\mathrm{H}$, Gutzmer $\mathrm{R}$, Choritz $\mathrm{H}$, et al. Life expectancy in primary myelodysplastic syndromes: a prognostic score based upon histopathology from bone marrow biopsies of 569 patients. Eur J Haematol 1994;53:280-287.

9 Gianelli U, Vener C, Bossi A, et al. The European Consensus on grading of bone marrow fibrosis allows a better prognostication of patients with primary myelofibrosis. Mod Pathol 2012;25:1193-1202.

10 Greenberg PL, Tuechler H, Schanz J, et al. Revised international prognostic scoring system for myelodysplastic syndromes. Blood 2012;120:2454-2465.

11 Schanz J, Tuchler H, Sole F, et al. New comprehensive cytogenetic scoring system for primary myelodysplastic syndromes (MDS) and oligoblastic acute myeloid leukemia after MDS derived from an international database merge. J Clin Oncol 2012;30:820-829.

12 Thiele J, Kvasnicka HM, Facchetti F, et al. European consensus on grading bone marrow fibrosis and assessment of cellularity. Haematologica 2005;90: 1128-1132.

13 Khoury JD, Sen F, Abruzzo LV, et al. Cytogenetic findings in blastoid mantle cell lymphoma. Hum Pathol 2003;34:1022-1029.

14 Chen W, Konoplev S, Medeiros LJ, et al. Cuplike nuclei (prominent nuclear invaginations) in acute myeloid leukemia are highly associated with FLT3 internal tandem duplication and NPM1 mutation. Cancer 2009;115:5481-5489.

15 Millecker L, Lennon PA, Verstovsek S, et al. Distinct patterns of cytogenetic and clinical progression in chronic myeloproliferative neoplasms with or without JAK2 or MPL mutations. Cancer Genet Cytogenet 2010;197:1-7.

16 Gustafson SA, Lin P, Chen SS, et al. Therapy-related acute myeloid leukemia with $t(8 ; 21)$ (q22;q22) shares many features with de novo acute myeloid leukemia with $\mathrm{t}(8 ; 21)(\mathrm{q} 22 ; \mathrm{q} 22)$ but does not have a favorable outcome. Am J Clin Pathol 2009;131:647-655.
17 Tefferi A. Pathogenesis of myelofibrosis with myeloid metaplasia. J Clin Oncol 2005;23:8520-8530.

18 Buhr T, Busche G, Choritz H, et al. Evolution of myelofibrosis in chronic idiopathic myelofibrosis as evidenced in sequential bone marrow biopsy specimens. Am J Clin Pathol 2003;119:152-158.

19 Kroger N, Zabelina T, van Biezen A, et al. Allogeneic stem cell transplantation for myelodysplastic syndromes with bone marrow fibrosis. Haematologica 2011;96:291-297.

20 Kroger N, Kvasnicka M, Thiele J. Replacement of hematopoietic system by allogeneic stem cell transplantation in myelofibrosis patients induces rapid regression of bone marrow fibrosis. Fibrogenesis Tissue Repair 2012;5(Suppl 1):S25.

21 Ohyashiki K, Aota Y, Akahane D, et al. The JAK2 V617F tyrosine kinase mutation in myelodysplastic syndromes (MDS) developing myelofibrosis indicates the myeloproliferative nature in a subset of MDS patients. Leukemia 2005;19:2359-2360.

22 Olsen RJ, Dunphy CH, O’Malley DP, et al. The implication of identifying JAK2 ( V617F ) in myeloproliferative neoplasms and myelodysplastic syndromes with bone marrow fibrosis. J Hematop 2008;1:111-117.

23 Yip SF, So CC, Chan AY, et al. The lack of association between JAK2 V617F mutation and myelodysplastic syndrome with or without myelofibrosis. Leukemia 2006;20:1165.

24 Kremer M, Horn T, Dechow T, et al. The JAK2 V617F mutation occurs frequently in myelodysplastic/myeloproliferative diseases, but is absent in true myelodysplastic syndromes with fibrosis. Leukemia 2006; 20:1315-1316.

$25 \mathrm{Fu}$ B, Ok CY, Goswami M, et al. The clinical importance of moderate/severe bone marrow fibrosis in patients with therapy-related myelodysplastic syndromes. Ann Hematol 2013;92:1335-1343.

26 Valent P, Orazi A, Busche G, et al. Standards and impact of hematopathology in myelodysplastic syndromes (MDS). Oncotarget 2010;1:483-496.

27 Sokol L, Caceres G, Rocha K, et al. JAK2(V617F) mutation in myelodysplastic syndrome (MDS) with del(5q) arises in genetically discordant clones. Leuk Res 2010;34:821-823.

28 Mesa RA, Silverstein MN, Jacobsen SJ, et al. Population-based incidence and survival figures in essential thrombocythemia and agnogenic myeloid metaplasia: an Olmsted County Study, 1976-1995. Am J Hematol 1999;61:10-15. 